\title{
A GEM-TPC Detector for FOPI and PANDA
}

\author{
Martin Berger ${ }^{* \dagger}$ \\ TUM \\ E-mail: mbergereph.tum.de
}

\begin{abstract}
A Time-Projection-Chamber (TPC) allows high-precision 3-dimensional tracking of charged particles in large volumes together with excellent particle identification capabilities. Up to now it was not possible to operate a TPC in a continuous mode due to back drifting secondary ions from the amplification stage. We want to overcome this limitation by using GEM foils for the amplification taking advantage of the intrinsic ion backflow suppression of the GEM foils.

Such a continuously readout TPC is one option for the inner tracker of the future PANDA experiment which will be built at the new Facility for Antiproton and Ion Research (FAIR) at Darmstadt, Germany. To verify the feasibility of this solution, a prototype detector with a diameter of $300 \mathrm{~mm}$ and a drift length of $730 \mathrm{~mm}$ was built and tested inside the FOPI at GSI for the first time in November 2010. The charge signal is amplified by a triple GEM stack and detected by 10254 pads of hexagonal shape with an outer diameter of $1.5 \mathrm{~mm}$. The hexagonal shape of the readout pads is expected to provide a more uniform charge distribution across the pads. The readout of the TPC is realized by an analog sampling of the signal with the AFTER chip [5], and subsequent digitization by a pipelined ADC.
\end{abstract}

XLIX International Winter Meeting on Nuclear Physics

24-28 January 2011

BORMIO, Italy

* Speaker.

${ }^{\dagger}$ For the GEM-TPC Collaboration 


\section{Introduction}

To face the challenges of the physics program in $\overline{\mathrm{P}} \mathrm{ANDA}^{1}$, the cylindrical central tracker of the Target Spectrometer has to fulfill the following requirements [1]: A high vertex resolution $\left(\sigma_{r \varphi} \approx 150 \mu \mathrm{m}, \sigma_{z} \approx 1 \mathrm{~mm}\right)$, high momentum resolution $(\approx 1 \%)$, minimal material budget $(\approx 1 \%$ of radiation length), high rate capability, resistance against aging, etc. Due to the beam characteristics $\left(\mathscr{L}=2 \cdot 10^{32} \mathrm{~m}^{-2} \mathrm{~s}^{-1}, 2 \cdot 10^{7} \bar{p} p\right.$ annihilations $\left.\mathrm{s}^{-1}\right)$, the TPC has to work in a continuous mode, i.e. without gating, which is another big challenge from the technical point of view. Due to the rather long electron drift time, tracks from up to 1000 events are superimposed inside the TPC at any given time.

A Time Projection Chamber with Gas Electron Multiplier readout not only fulfills all the requirements above, it furthermore provides very good $\mathrm{dE} / \mathrm{dx}$ measurement also in the region of low momenta, which is necessary for particle identification.

In addition, the successful long-term operation of GEM-Detectors for example at the COMPASS ${ }^{2}$ experiment[2] shows that this kind of detector has excellent properties concerning high rate capability. The GEM detectors have due to the asymmetric field configuration a high intrinsic suppression of ion back flow [7]. Such properties enables a GEM-based TPC to operate in an ungated mode. In the next sections the present status of the development, the progress and test results of a GEM-TPC prototype [3] designed and built by a collaboration of groups from GSI Darmstadt, HISKP Bonn, SMI Vienna, and TU München will be discussed in detail.

This prototype was build to fit into the FOPI spectrometer at GSI were it is very useful to increase primary and secondary vertex resolutions. Together with the central drift chamber and the time of flight detectors of FOPI the reconstruction of strange particles like $\mathrm{K}^{+}, \mathrm{K}^{-}, \phi, \mathrm{K}_{s}^{0}$ and $\Lambda$ will be improved significantly and the phase space coverage will be increased.

\section{The Prototype Detector}

The GEM-TPC prototype chamber has a total drift length of $727.8 \mathrm{~mm}$, an inner diameter of $104 \mathrm{~mm}$ and an outer diameter of $308 \mathrm{~mm}$. The prototype mainly consists of three parts: the fieldcage, the readout part and the media flange. Figure 1 shows an explosion view of the GEM-TPC prototype

\subsection{The Fieldcage}

The lightweight field-cage structure is made out of a composite material, containing mainly Kapton and Rohacell. Materials of the fieldcage and their thickness is listed in table 1.

The inner part of the field-cage contains the strip-foil to homogenize the electric field of the lateral area. The stripfoil is made out of a $25 \mu \mathrm{m}$ thick kapton foil with over 700 copper strips with a pitch of $1.5 \mathrm{~mm}$ on each sides. The radiation length for a track perpendicular to the beam axis is $0.6 \%$ and for a track in forward direction, crossing the drift cathode, it is $0.6 \%$.

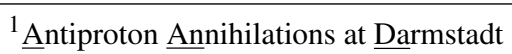

${ }^{2}$ Common Muon and Proton Apparatus for Structure and $\underline{S}$ pectroscopy
} 


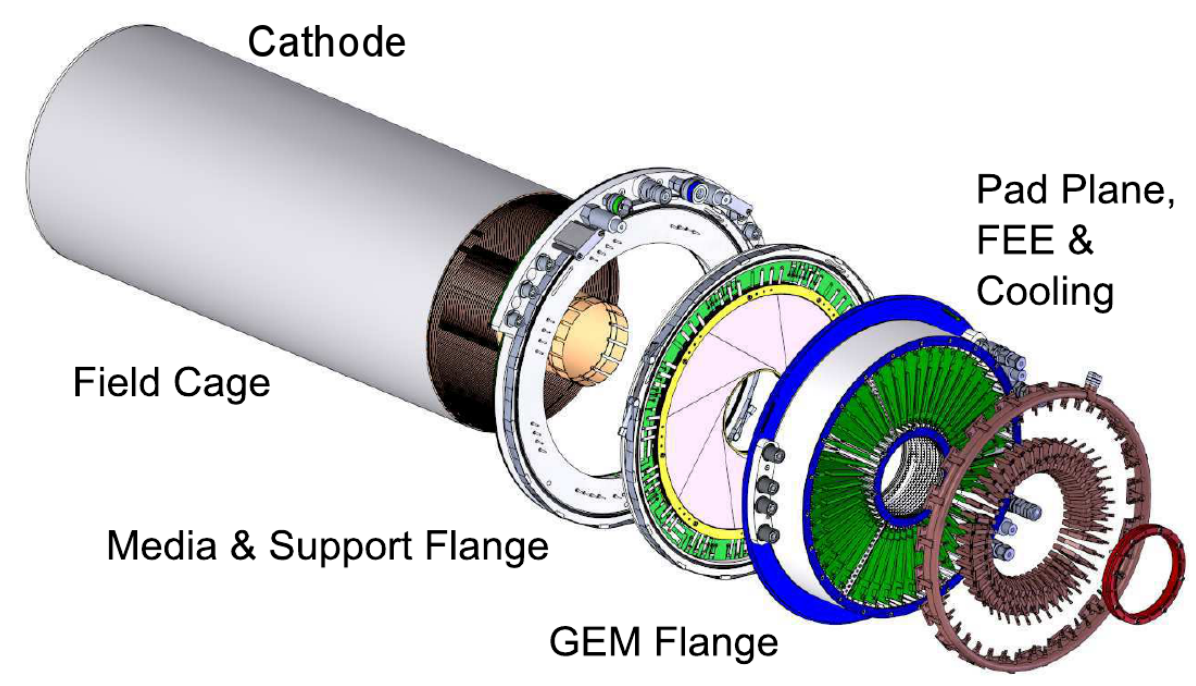

Figure 1: Explosion view of the GEM-TPC.

\begin{tabular}{|c|c|}
\hline Material & Thickness \\
\hline Aluminized mylar & $200 \mathrm{~nm}$ \\
\hline Kapton & $25 \mu \mathrm{m}$ \\
\hline Kapton & $125 \mu \mathrm{m}$ \\
\hline Rohacell & $2 \mathrm{~mm}$ \\
\hline Kapton & $125 \mu \mathrm{m}$ \\
\hline Rohacell & $2 \mathrm{~mm}$ \\
\hline Kapton & $125 \mu \mathrm{m}$ \\
\hline Kapton & $25 \mu \mathrm{m}$ \\
\hline
\end{tabular}

Table 1: Materials and their thickness listed from the outside of the fieldcage to the inside.

\subsection{The GEM Flange}

The GEM-Flange is designed to hold up to four GEM foils for the gas amplification. The foils used for the prototype are standard foils produced at CERN with an thickness of $50 \mu \mathrm{m}$ and a hole pitch of $140 \mu \mathrm{m}$ To keep the charge released in a discharge as low as possible each foil is segmented into 8 sectors on one side each with its own loading resistor. A schematic of the GEM-stack how it is used is visible in Figure 2.

\subsection{The Readout}

For the readout the padplane with 10254 hexagonal pads is equipped with 42 front-end cards, each with 4 T2K/AFTER chips [5]. The T2K/AFTER chip is an analog sampling chip with a 511cell FIFO memory per channel, a sampling frequency of $16 \mathrm{MHz}$ or $20 \mathrm{MHz}$ and a multiplexed output. The design value of the equivalent noise charge (ENC) is around 600 electrons at a $10 \mathrm{pF}$ input capacitance. Picture 3 shows the 2-dimensional noise distribution on the padplane. One can see that the noise is rather uniform and around 1.2 ADC channels which corresponds to an ENC of 625 electrons. The visible ring structure is due to channels with longer tracks on the outer parts of 


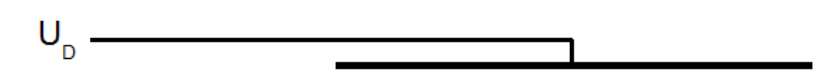

$727.80 \mathrm{~mm}$

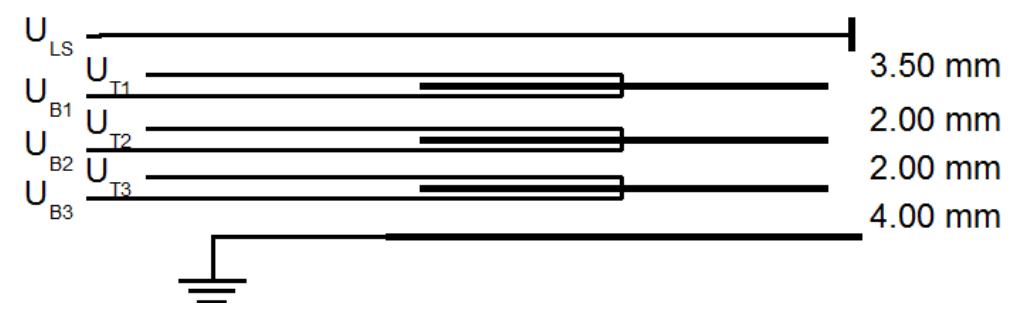

Figure 2: Scheme of the GEM-stack how it is used in the prototype where $U_{D}$ is the drift voltage, $U_{L S}$ is the voltage of the las strip before the GEM foils and $U_{T}$ and $U_{B}$ are the top and bottom voltages of the respective GEM foil.

the front-end PCB.

Since the drift velocity depends strongly on the temperature, a water-driven cooling system for the front-end cards was installed to avoid heating of the padplane and consequently a local heating of the gas. Twelve PT-100 temperature sensors were installed on the padplane to monitor the stability of the temperature. The cooling system is also used to keep the ADC's at a constant temperature.

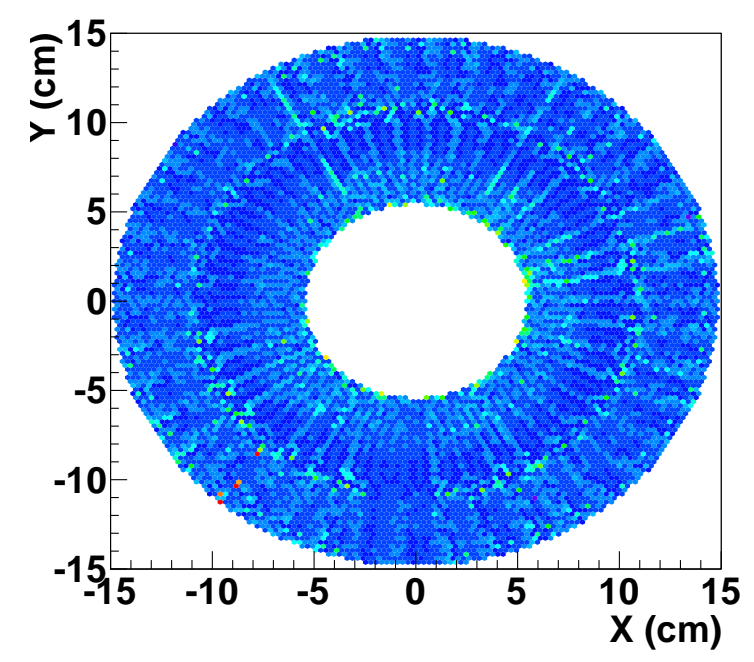

Figure 3: 2-Dimensional noise distribution on the padplane.

\section{First test inside FOPI}

To test the operation of the prototype it was installed inside the FOPI [4] spectrometer at GSI (Darmstadt, Germany). In Figure 4 FOPI (grey color) as well as the GEM-TPC (violet color) are shown. The FOPI spectrometer consists of a central drift chamber (CDC) which is surrounded by 
a scintillator barrel (Barrel) and an RPC barrel (RPC) with an intrinsic time of flight resolution of 80 ps. In forward direction another drift chamber (Helitron) and a scintillator time of flight wall (PLAWA) with a time of flight resolution of $120 \mathrm{ps}-150 \mathrm{ps}$ are installed. The CDC, Helitron and the two barrels are surrounded by a $0.6 \mathrm{~T}$ solenoid magnet (not shown in Fig. 4). The TPC was mounted in the inner hole of the central drift chamber of FOPI. With this configuration it is possible to use the detectors of FOPI as a reference. All detectors of FOPI have a $2 \pi$ acceptance in the polar

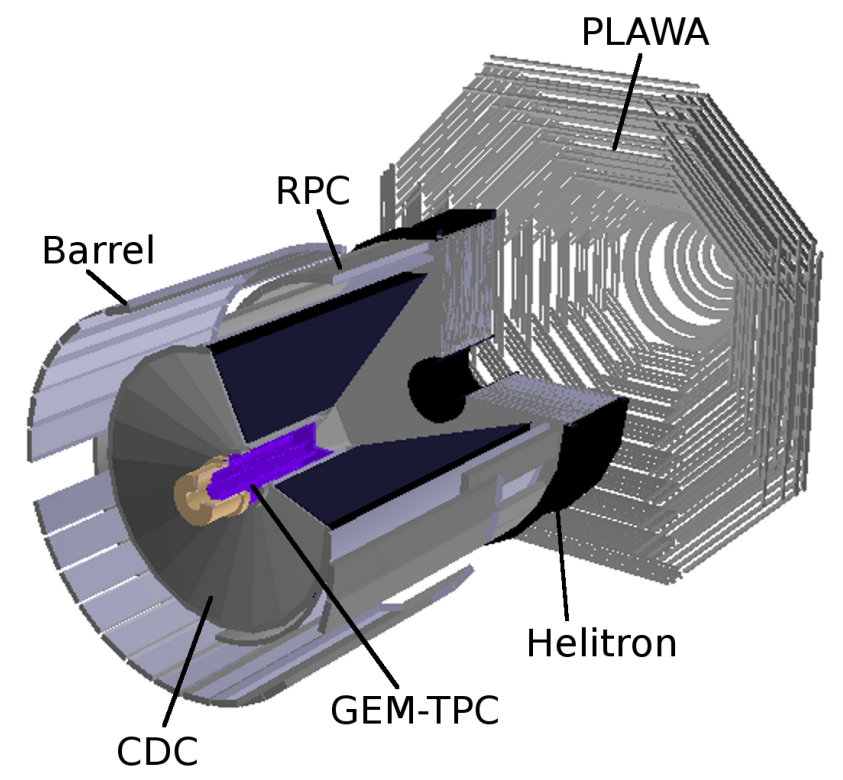

Figure 4: The FOPI spectrometer (gray color) with the GEM-TPC (violet color) prototype mounted in the inner bore of the central drift chamber (CDC).

angle. The acceptance in the azimuthal angles are listed in table 2. The FOPI spectrometer has

\begin{tabular}{|c|c|}
\hline Detector & Acceptance \\
\hline CDC & $30^{\circ}-140^{\circ}$ \\
\hline RPC & $36^{\circ}-67^{\circ}$ \\
\hline Helitron & $10^{\circ}-30^{\circ}$ \\
\hline Barrel & $60^{\circ}-110^{\circ}$ \\
\hline PLAWA & $10^{\circ}-35^{\circ}$ \\
\hline GEM-TPC & $5^{\circ}-175^{\circ}$ \\
\hline
\end{tabular}

Table 2: Acceptances of the FOPI detectors and the GEM-TPC.

vertex resolution of few millimeters in the $x-y$ plane and a resolution along the beam axis of around $15 \mathrm{~cm}$. Vertex and especially secondary vertex resolutions can be significantly improved by using the GEM-TPC prototype as an additional tracker.

\subsection{Data acquisition}

The data acquisition (DAQ) of FOPI is based on the Multi Branch System (MBS) and designed for a trigger-rate of around $100 \mathrm{~Hz}$. The DAQ of the GEM-TPC is based on a different system since 
the VME readout like in FOPI would be to slow to handle the large amount of data coming from the TPC ( $40 \mathrm{MB} / \mathrm{s})$. This system is a slightly changed version of the COMPASS readout [8]. A way to merge the data streams of both systems had to be found. The trigger, generated by the FOPI system is sent to the Trigger Control System (TCS) were it is distributed to the TPC readout. The deadtime of around $2.5 \mathrm{~ms}$ generated by this system is merged with the FOPI deadtime. Optical fibers are used to send the data of the prototype via the S-Link protocol [8] to the eventbuilder were it is merged with the FOPI data stream. The merging is done by using using unique event numbers or timestamps which were distributed to both data streams before.

Furthermore a lot of different programs to monitor and control the high voltage, low voltage, temperatures, gas mixture, DAQ and data were developed and improved.

\subsection{Tests with Cosmics inside FOPI}

As a first test, tracks from cosmic rays were measured, using the Barrel was as a trigger. The TPC was operated at a drift-field of $360 \mathrm{~V} / \mathrm{cm}$ and a $\mathrm{Ar} / \mathrm{CO}_{2}$ drift gas in a 90/10 mixture by volume which translates into a drift velocity of $2.9 \mathrm{~cm} / \mu \mathrm{s}$. The track reconstruction was done by first finding the track points with a 3D clustering algorithm. The obtained cluster are used in a 4D Hough transformation for linear straight line pattern recognition. The residuals Hough transformation for tracking are shown in Figure 5. A spatial resolution of $230 \mu \mathrm{m}$ for the first slice along the z-axis was obtained.

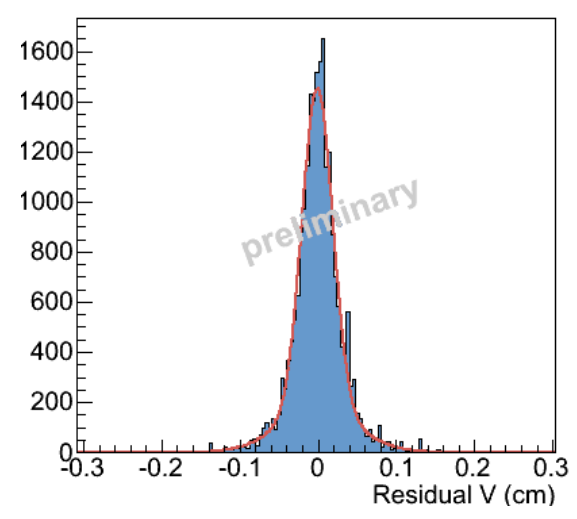

(a) Residual with two Gaussian curves fitted, measured with cosmics. The weighted mean of the sigmas of the Gaussian curves is $230 \mu \mathrm{m}$.

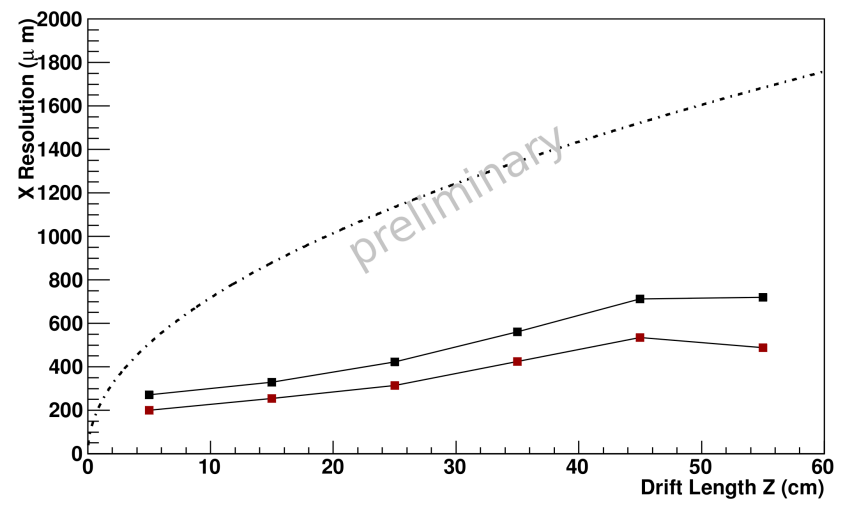

(b) The dashed line is the transverse diffusion for single electron drift as calculated with GARFIELD. The dots show the results from residual distributions for tracks from $10 \mathrm{~cm}$ bins along the drift direction, as seen in the left plot. The red dots are the sigmas of the narrow Gaussian, while the black dots above are from the weighted mean of both Gaussian curves.

Figure 5: Picture (a) shows the residuals of one slice in z. Picture (b) shows the z-dependency of the residuals.

\subsection{Beam test}

During the first test beamtime from in November 2010 and April 2011 data with different settings were collected. 
- Several drift fields: $150,200,300,360 \mathrm{~V} / \mathrm{cm}$

- With corresponding drift velocities: 0.9, 1.4,2.2,2.9 cm/ $/ \mu$ [6]

- Different settings for the gain for the GEM-stack from 2000 to 5000

- Two gas mixtures, namely $\mathrm{Ar} / \mathrm{CO}_{2}$ and $\mathrm{Ne} / \mathrm{CO}_{2}$ both in a $90 / 10$ mixture by volume

- 84-Kr, 197-Au and 22-Ne beams at 1.2 AGeV, 1.0 AGeV and 1.7 AGev kinetic energies

The chamber also performed well with beam and tracks were visible as one can see in Figure 6. It was also possible to do a matching between the CDC and the TPC. A matched track is shown in Figure 7
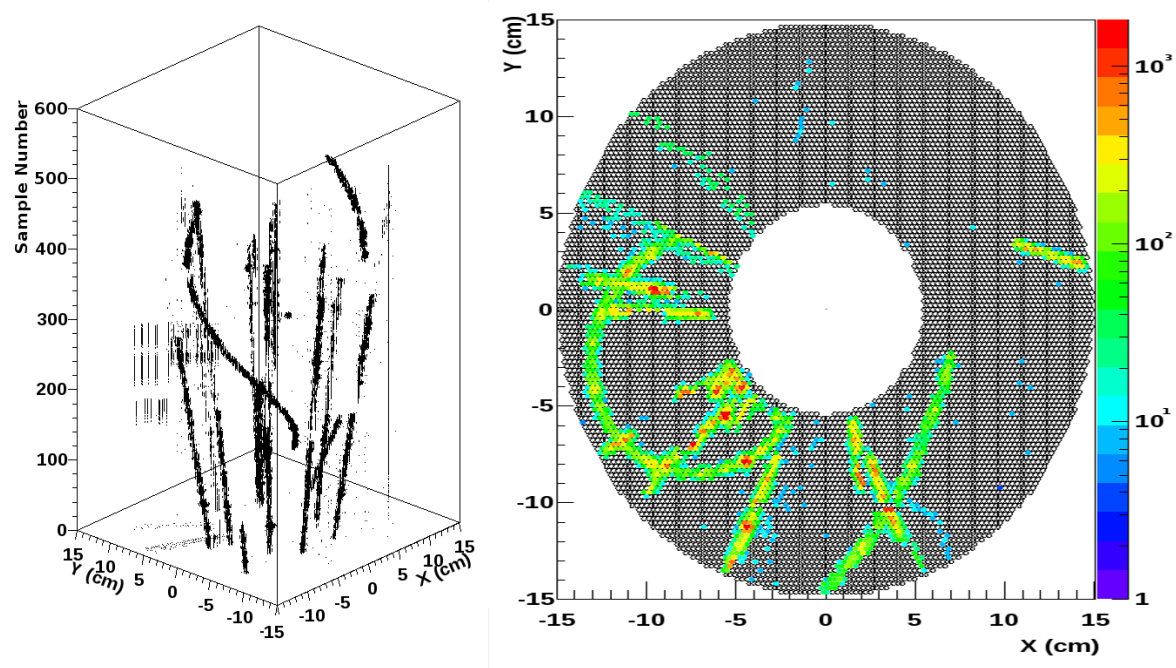

Figure 6: A typical event in the GEM-TPC. The left panel shows the samples from the ADC in both padplane position and sample time while the right picture shows the projection onto the padplane. The color coding corresponds to the amplitude of a signal.

\section{Summary and Outlook}

A large size GEM-TPC prototype was designed and built. It was fully equipped with front-end electronics for more than 10000 channels. The detector was installed inside FOPI and commissioned with cosmics where a resolution if $230 \mu \mathrm{m}$ was obtained. A successful test with heavy ion reactions and a measure campaign with different gain, gas and drift-field settings was done. The evaluation of the collected data is ongoing. The next step is the combined analysis of FOPI CDS and GEM-TPC prototype data. A physics run with pion-induced reactions will be done in June 2011. 


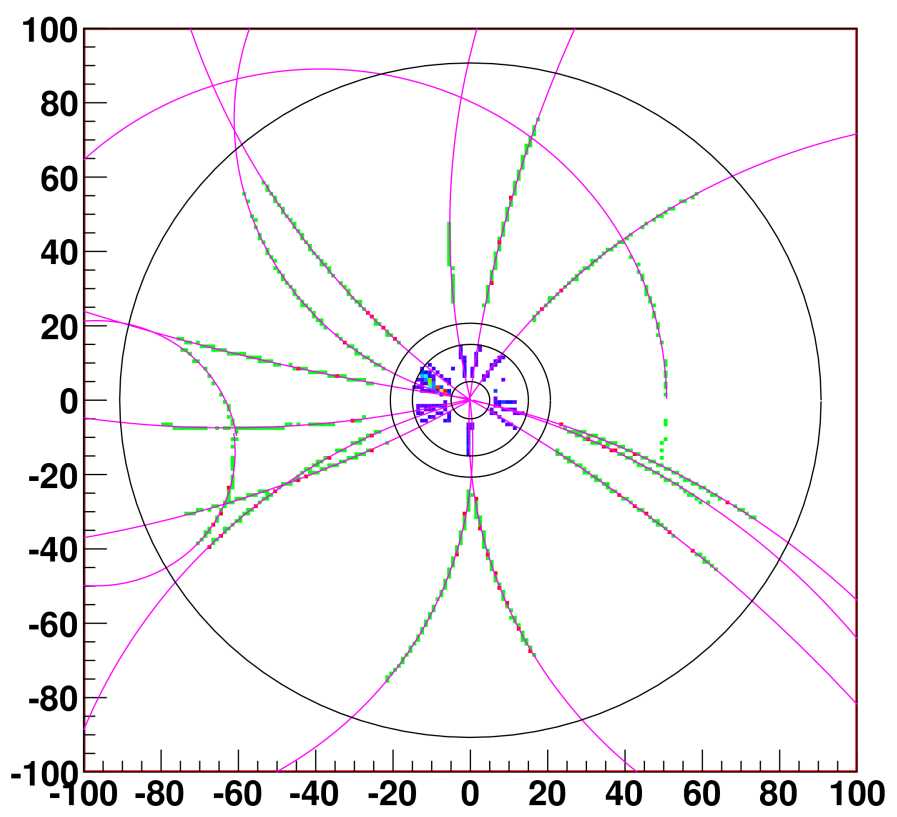

Figure 7: A matched event in TPC (two inner circles) and CDC (two outer circles) in a x-y projection. The pink lines indicate the reconstructed tracks of the CDC while the green point are CDC hit points and the blue points are hits in the TPC

\section{References}

[1] The PANDA Collaboration, GSI, 2005, http: Ilwww.gsi.de.

[2] B. Ketzer, et al., Nucl. Instr. and Meth. A, 535 (2004) 314-318.

[3] L. Fabbietti, et al., Nucl. Instr. and Meth. A, 628 (2011) 204-208.

[4] J. Ritman, et al., Nucl.Phys.Proc.Suppl. 44(1995) 708-715.

[5] P. Baron et al., IEEE Trans. Nucl. Sci. NS-55 (2008) 1744.

[6] T. Zhao, et al., Nucl. Instr. and Meth. in Phys. Res. A. 340 (1994) 485-490.

[7] M. Killenberg et al., Nucl. Instr. and Meth. A498 (2003) 269.

[8] L. Schmitt, et al.: IEEE Transactions on Nuclear Science 51 (2004) 439-444. 